\title{
Self-tuning dead beat PD controller for pitch angle control of a bench-top helicopter
}

\begin{abstract}
This paper presents an improved robust Proportional Derivative controller for a 3-Degree-ofFreedom (3-DOF) bench-top helicopter by using adaptive methodology. Bench-top helicopter is a laboratory scale helicopter used for experimental purposes which is widely used in teaching laboratory and research. Proportional Derivative controller has been developed for a 3-DOF bench-top helicopter by Quanser. Experiments showed that the transient response of designed PD controller has very large steady state error i.e., 50\%, which is very serious. The objective of this research is to improve the performance of existing pitch angle control of PD controller on the bench-top helicopter by integration of PD controller with adaptive controller. Usually standard adaptive controller will produce zero steady state error, however response time to reach desired set point is large. Therefore, this paper proposed an adaptive with deadbeat algorithm to overcome the limitations. The output response that is fast, robust and updated online is expected. Performance comparisons have been performed between the proposed self-tuning deadbeat PD controller and standard PD controller. The efficiency of the self-tuning dead beat controller has been proven from the tests results in terms of faster settling time, zero steady state error and capability of the controller to be updated online.
\end{abstract}

Keyword: Adaptive control; Deadbeat control; Bench-top helicopter; Self-tuning control 